\title{
Rapid Fourier Masked Domain Mapping to Reveal Head to Head Charged Domain Walls in Lead Titanate
}

\author{
K. Moore ${ }^{1}$, U. Bangert ${ }^{1}$, M. Conroy ${ }^{1}$, E. O'Connell ${ }^{1}$ and J.M. Gregg ${ }^{2}$ \\ 1. Department of Physics, University of Limerick, Limerick, Ireland \\ 2. Centre for Nanostructured Media, Institute of Physics, Queen's University Belfast, Northern Ireland, \\ $\mathrm{UK}$ \\ * Corresponding author: kalani.moore@ul.ie
}

Domain walls (DWs) in ferroelectrics have attracted widespread in recent years as they offer 2D moveable and controllable features with tunable properties, suggesting many possible uses in future nanoelectronics. Charged and conducting DWs in particular are rich areas in exploring fundamental physics of many materials $[1,2]$. One widely explored and utilised class of these are ferroelectric perovskites. Lead titanate, $\mathrm{PbTiO}_{3}$ is of this family but is traditionally challenging to produce in its pure form owing to the large c/a ratio producing significant stress in any crystal grown [3].

Here single the multi domain state of crystal lead titanate (PTO) is characterised using high resolution transmission electron microscopy (HR-TEM). Lamallae were ion sliced from different areas of the crystal and the different domain orientations were rapidly mapping using a technique of fourier masking to determine the polarisation axes.

Following this, interesting regions were identified and investigated on the atomic scale using scanning transmission electron microscopy (STEM), electron energy loss spectroscopy (EELS) and geometric phase analysis (GPA). The results are discussed and the general identification and orientation of ferroelectric domain superlattices in bulk crystals and lamellae are presented.

The domain mapping combined with knowledge of domain formation in PTO showed unusual junctions exhibiting higher contrast in STEM were indeed charged. Subsequent Ti shift mapping and comparison to the literature shows that these DWs were 180 degree head to head type and likely metallic in nature [4, 5]. The energetics behind the formation of the charged junctions are discussed, the influence of flexoelectricity is introduced with effects on the wider domain structure demonstrated [6].

These results represent the first 180 degree head to head charged DWs in PTO investigated at the atomic level. They lend valuable insight into the prospect of strain engineering useful 2D features in ferroelectric perovskites mediated by flexoelectricity. They also offer give important knowledge in resolving domains structures and characteristic features to identify charged and conducting DWs with atomic resolution STEM.

\section{References:}

[1] H Wu et al., J. Eur. Ceram. Soc. 35 (2015), p. 411.

[2] G. Catalan et al., "Domain wall nanoelectronics," 2012.

[3] VA Chaudhari and GK Bichile, Smart Mater. Res. 2013 (2013), p. 1.

[4] MY Gureev, AK Tagantsev, and N Setter, Phys. Rev. B 83 (2011), p. 184104.

[5] YL Tang et al., Science, 348 (2015), p. 547. 
[6] AK Tagantsev, "Piezoelectricity and flexoelectricity in crystalline dielectrics," 1986.

B

A1

$\mathrm{A} 2$

A1

A2

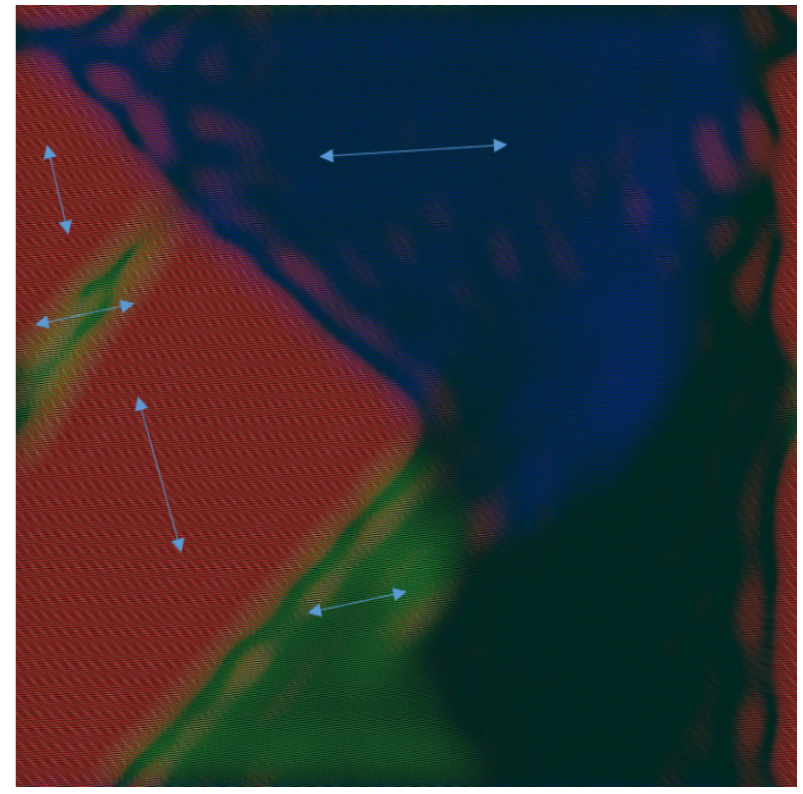

Figure 1. Left, STEM image of ferroelectric domains in PTO with the charged DW highlighted. Right, polarisation axes mapped by fourier masking of the STEM image. 